\title{
Rapport statutaire 2002 de la FMH
}

\section{Table des matières}

\section{Avant-propos}

\section{Organes de la Fédération}

1. Chambre médicale

2. Conférence des présidents

3. Commission des finances

4. Conférence pour la formation postgraduée et continue

5. Comité central

6. Conseil suisse de déontologie

\section{Message du Président}

\section{Rapports des membres du Comité central}

1. Dr Hans Heinrich Brunner, président de la FMH

2. Dr Ursula Steiner-König, vice-présidente de la FMH

3. Dr Yves Guisan, vice-président de la FMH

4. Dr Max Giger

5. Dr Claude Aubert

6. Dr Ludwig T. Heuss

7. Dr Reto Laetsch

8. Dr Marie-Christine Peter

9. Pr Verena Briner

10. Dr Franco Muggli

11. Dr Susi Stöhr

\section{Relations internationales}

1. Comité Permanent des Médecins Européens (CPME) / Conférence Européenne des Ordres des Médecins (CEOM)

2. Union Européenne des Médecins Omnipraticiens (UEMO)

3. Union Européenne des Médecins Spécialistes (UEMS)

4. Association Européenne des Médecins des Hôpitaux (AEMH)

5. European Forum of Medical Associations and WHO (EFMA / WHO)

6. World Medical Association (WMA)

\section{Secrétariat général / comptes annuels}

1. Quelle année!

2. Personnel

3. Bilan

4. Compte d'exploitation 


\section{Avant-propos}

Chers membres de la FMH,

chères lectrices et chers lecteurs,

Le Comité central de la FMH a décidé, début 2003, de diviser cette année le rapport annuel de la Fédération en trois parties:

1. rapport statutaire des organes de la FMH;

2. rapport de gestion du Secrétariat général;

3. présentation et rapport d'activité des divers services et domaines.

Pourquoi cette nouvelle articulation? La présentation adoptée jusqu'ici paraissait quelque peu dépassée et l'on souhaitait introduire une formule plus moderne, mieux structurée et au graphisme attrayant. Ce faisant, il s'agissait bien sûr de préserver les dispositions statutaires ainsi que l'intérêt légitime de nos membres à obtenir des informations détaillées.

Dès lors, où trouver quelles informations? Comme jusqu'ici et conformément aux statuts de la $\mathrm{FMH}$, le rapport annuel est publié dans le Bulletin des médecins suisses. Ce rapport dit «statutaire», que vous tenez en mains, rassemble les comptes rendus des organes de la Fédération (Chambre médicale, Conférence des présidents, Commission pour la formation postgraduée et continue, etc.). La Chambre médicale se fonde sur ce document et sur les comptes annuels pour donner décharge au Comité.
Quant au rapport statutaire que vous trouverez en annexe à la présente édition du Bulletin des médecins suisses, il constitue un nouvel outil de communication. Outre des données structurelles et financières sur la FMH et son Secrétariat général, il comprend de brefs commentaires sur les grands thèmes qui ont marqué l'année écoulée. La FMH destine cette publication non seulement à ses membres, mais aussi au grand public qu'elle entend informer de ses activités et de ses prestations.

Finalement, dans un troisième volet conçu lui aussi comme un instrument de communication et qui sera publié par tranches à intervalles réguliers dans le Bulletin, les divers domaines et services d'état-major du Secrétariat général tenteront de vous donner une image détaillée de leur activité.

Nous espérons que ces innovations en matière de politique d'information correspondront à vos attentes et vous aideront à garder le cap dans l'univers de plus en plus déroutant de la politique professionnelle. Et comme toujours lorsque des nouveautés sont introduites, toute suggestion ou critique de votre part sera la bienvenue.

Annamaria Müller Imboden, secrétaire générale

Dr Markus Trutmann, rédacteur en chef du Bulletin des médecins suisses 


\section{Organes de la Fédération}

\section{Chambre médicale}

Pendant l'année sous revue, la Chambre médicale ne s'est réunie qu'une seule fois. Cette séance ordinaire s'est à nouveau tenue relativement tôt dans l'année, le 25 avril 2002, à un endroit facilement accessible pour tous les délégués: le «Landhaus» de Soleure. Les adieux à FrançoisXavier Deschenaux en ont été un des points forts. Secrétaire général de la FMH pendant de longues années, il avait pris sa retraite à fin 2001 déjà. Un vibrant hommage lui a été rendu pour son engagement exceptionnel en faveur de la Fédération.

Après avoir accepté le rapport et les comptes annuels et octroyé la décharge au Comité central et au président, les délégués ont approuvé, non sans de longs débats, le budget 2003 qui prévoyait une hausse d'environ $10 \%$ des cotisations centrales. Ils ont ainsi posé un jalon pour assurer le financement de notre association professionnelle en cette période mouvementée pour le secteur de la santé publique. Le Dr Roland Schwarz, de Muttenz, a été élu à la commission des finances en remplacement du Dr Olivier Kappeler, membre sortant. Pour la première fois depuis longtemps, la Chambre médicale n'était pas placée sous le signe du TARMED, puisque seul le concept de remaniement (RE2) et son budget y ont été adoptés. On relèvera, toutefois, que le vœu du président de voir la question du TARMED réglée suite à la votation générale ne s'est pas réalisé pendant les mois qui ont suivi.

Un thème qui, en revanche, a donné lieu à d'intenses discussions à la Chambre était le comportement à adopter par la FMH au cas où le droit légal des médecins de facturer à la charge de l'assurance sociale serait aboli (levée de l'obligation de contracter). Il fut décidé de lancer un référendum si cette éventualité se matérialisait. S'agissant d'arrêter un mot d'ordre quant à la solution des délais, la Chambre médicale a rapidement atteint un consensus en faveur d'une dépénalisation de la démarche des femmes concernées. Les débats sur l'admission de différentes organisations de médecins ont été, par contre, des plus animés. Lors du vote, l'association des médecins de HMO et celle des sociétés de discipline non invasives (FMC) n'ont pas obtenu le droit d'in- tervention qu'elles sollicitaient et l'AMPHS n'a pas été reconnue comme organisation de base. Les délégués se sont montrés plus cléments envers la Société suisse des médecins-conseils (SSMC) et le Collège de médecine de premiers recours (CMPR), en leur accordant respectivement le droit d'intervention au sein des organes de la FMH et celui de siéger à la Commission de la formation postgraduée et continue (CFPC).

Annamaria Müller Imboden, secrétaire générale

\section{Conférence des présidents}

Si la première Conférence des présidents, tenue le 14 mars 2002, a surtout préparé la séance ordinaire de la Chambre médicale, les deux suivantes se sont penchées - les 5 septembre et 28 novembre 2002 - sur des questions fondamentales concernant le positionnement actuel et l'avenir du corps médical et de son association professionnelle. La nécessité d'un professionnalisme accru et d'un profil mieux affirmé a été mise en évidence. Les participants ont en outre exprimé leurs vives préoccupations face à l'attention pointilleuse et critique, voire soupçonneuse, dont leur corporation fait actuellement l'objet tant de la part du public que du monde politique et des autres acteurs du domaine de la santé. A cet égard, les irrégularités intervenues dans le décompte des prestations de laboratoire et le système d'appréciation des médecins proposé par Helsana - énergiquement contesté depuis par le préposé fédéral à la protection des données - ne constituent que la pointe de l'iceberg. Un enjeu beaucoup plus radical encore est et demeure la révision par étapes de la loi sur l'assurance-maladie (LAMal), y compris le projet de lever l'obligation de contracter. Quant à la décision de bloquer l'accès des jeunes médecins à la pratique privée, conçue en fait comme une manœuvre défensive pour prévenir une invasion de médecins venant de l'Union européenne, il paraît superflu de rappeler ici les déclarations chargées d'émotion qu'elle a suscitées.

Annamaria Müller Imboden, secrétaire générale 


\section{Commission des finances}

La Commission des finances, au sein de laquelle sont représentées (que les Romanches me pardonnent ...) les trois plus grandes régions linguistiques du pays, exerce une manière de contrôle politique sur les activités du Comité central en matière de finances. Loin d'être un organe de révision, elle donne son avis et émet des suggestions au sujet des divers postes de dépenses et de recettes.

Un règlement sur les sociétés participantes a ainsi été élaboré dans le courant de l'année écoulée sur recommandation de la commission. Il fixe des critères précis pour l'attitude à adopter par la FMH envers les sociétés anonymes ou les fondations, de même que pour sa représentation au sein de tels organismes. Il devrait permettre de prévenir à temps, et efficacement, des problèmes tels que ceux rencontrés avec la Centrale générale d'achat des médecins suisses (AESA) ou les Editions Huber.

C'est aussi sur l'insistance de la commission que la comptabilité analytique de la FMH, organisée par centres de charges, est devenue réalité.

En outre, toujours à l'initiative de la commission, l'abonnement de base au HIN a pu être mis à la disposition de tous les membres de la FMH.

Cependant, l'activité prioritaire de la Commission des finances consiste à contrôler les comptes et bilans de la FMH. Vu la nouvelle fonction déléguée à celle-ci en matière d'octroi des titres par le Département de l'intérieur, et si l'on songe au rôle primordial que joue la Réglementation pour la formation postgraduée dans la politique financière de la Fédération, cette tâche de contrôle revêt actuellement une importance toute particulière.

Enfin, cette année 2002 aura été marquée par le retrait du Dr Olivier Kappeler. Nous le remercions du travail qu'il a accompli au sein de la commission pendant de nombreuses années et souhaitons la bienvenue au Dr Roland Schwarz, élu par la Chambre médicale pour lui succéder.

Dr Adrian Sury, président, pour $l^{\prime}$ «Ordine dei medici del Canton Ticino», Dr Pierre Guillaume pour la Société romande de médecine,

Dr Roland Schwarz pour les sociétés de médecine de Suisse alémanique

\section{Commission pour la formation postgraduée et continue (CFPC)}

La CFPC est l'organe consultatif du Comité central et de la Chambre médicale pour toutes les questions touchant à la formation postgraduée et continue. Elle réunit des délégués de toutes les sociétés de discipline médicale ainsi que des facultés, de l'ASMAC, de l'AMPHS et des associations régionales (SMSR, VEDAG, OMCT). Une séance plénière, préparée par le comité de la CFPC qui comprend 19 membres, a lieu chaque année au printemps et en automne. Les membres du comité assument en outre des tâches importantes en qualité de «membres d'une autre discipline» au sein de la commission des titres et de celle des établissements de formation postgraduée, organes chargés respectivement de traiter les demandes de titres et de reconnaître les établissements de formation. Les affaires courantes sont expédiées par le bureau de la CFPC, constitué par le Dr Max Giger, président, et les Drs JeanPierre Keller et Susanna Stöhr, vice-présidents.

Pendant l'année écoulée, la CFPC s'est notamment penchée sur les questions suivantes:

- entrée en vigueur des accords bilatéraux et de la loi sur l'exercice des professions médicales et conséquences de cette mise en vigueur;

- introduction de concepts de formation postgraduée dans toutes les cliniques reconnues et réalisation des visites d'accréditation prévues;

- demandes de création ou de révision de différents programmes de formation postgraduée et attestations de formation complémentaire;

- promotion d'une formation en gestion pour tous les candidats à un titre de spécialiste;

- révision de la Réglementation pour la formation continue et définition des modalités d'exécution.

Christoph Hänggeli

\section{Comité central (CC)}

L'entrée en vigueur, le $1^{\text {er }}$ juin 2002, de la loi fédérale révisée sur l'exercice des professions médicales (LEPM) a entraîné un changement de statut décisif pour la FMH. D'entité suprême à laquelle il fallait presque obligatoirement adhérer, elle est devenue une association professionnelle dont on peut ou non devenir membre. Même s'il n'en a pas résulté un exode de membres comme on l'avait craint tout d'abord, la Fédération et son Comité central se sont vus et se voient en- 
core amenés à mettre en place dans l'urgence des stratégies et des mesures visant à rendre une adhésion attrayante. Le CC s'est donc retiré à plusieurs reprises pour des journées de réflexion au cours desquelles, en petits groupes et en s'isolant parfois du monde, il s'est attelé à «forger» un nouvel avenir pour le corps médical en général et pour son association professionnelle en particulier. Même s'il n'a pas encore - on s'en doute - trouvé l'œuf de Colomb, il a néanmoins mis en route un processus pragmatique de réorientation de la $\mathrm{FMH}$, qui se poursuivra par étapes. Bien qu'un tel processus, comme l'ont montré les débats lors des diverses rencontres statutaires, ne puisse se dérouler sans douleurs ni conflits, un premier pas significatif dans une nouvelle direction a été franchi pendant l'année sous revue.

Les séances ordinaires du Comité central faisant l'objet de comptes rendus réguliers dans le Bulletin des médecins suisses, nous renonçons à évoquer ici le détail des thèmes traités et des décisions prises.

Annamaria Müller Imboden, secrétaire générale

\section{Conseil suisse de déontologie (CSD)}

En 2002, dix cas de litiges ont été traités par le Conseil suisse de déontologie. Tous les recours ont pu être conclus par une décision ou une décision de classement. Cinq de ces plaintes se sont vues rejetées pour des raisons formelles après le premier échange d'écritures. Le règlement du CSD fixe les conditions à remplir pour qu'un recours puisse être traité sur le fond. En principe, seuls les membres FMH ont droit à saisir le Conseil suisse de déontologie et ils doivent s'acquitter d'une avance sur les coûts de procédure immédiatement après l'ouverture de celle-ci.
Dans les cinq cas précités, ces conditions n'étaient pas remplies ou il n'existait pas de décision de première instance susceptible d'être contestée.

Dans cinq autres cas, l'échange d'écritures et la procédure probatoire ont eu lieu et, les tentatives de conciliation ayant échoué, une décision a été prise. Il est arrivé à quelques reprises que, suite aux conseils prodigués en matière de procédure, d'interprétation et d'application du code de déontologie, ou à la clarification de la décision de première instance, les personnes concernées renoncent à introduire leur recours.

Une partie des recours interjetés pendant la période sous revue concernaient l'application des dispositions en matière d'utilisation des titres de formation postgraduée, ainsi que la présentation et le contenu d'annonces dans les médias: une publicité non conforme à la déontologie, liée au non-respect des prescriptions contre la concurrence déloyale, a poussé les collègues ou la société cantonale concernés à intervenir. La violation du secret professionnel, le refus de livrer un dossier médical ou sa livraison tardive, le caractère non économique de procédures de comptabilisation et de facturation ainsi qu'un comportement contraire à la déontologie vis-à-vis de confrères ou de patients ont constitué d'autres causes de recours.

Lors de sa séance annuelle, le bureau du CSD, constitué par le président et les deux vice-présidents, s'est prononcé en faveur de l'élaboration d'une banque de données qui permettra d'archiver les décisions du CSD et celles des commissions de déontologie des sociétés cantonales de médecine. La collecte sous forme électronique de décisions anonymisées devrait permettre d'unifier l'application et l'exécution du code de déontologie de la FMH.

Dania Ischi-Ceppi 


\section{Message du Président}

\section{Si tu veux la paix, prépare la guerre}

L'événement sans doute le plus important de l'année sous revue a été la votation générale sur le TARMED, objet qui fut clairement approuvé. Il serait bon que tous les médecins acceptent ce vote selon les règles de bienséance qui sont celles de notre pays, à savoir comme le résultat d'une démarche parfaitement démocratique. Il semble que pour certains groupes d'intérêts, cela soit particulièrement difficile à admettre.

Nous le savons tous, il reste encore fort à faire avant de pouvoir appliquer cette décision avec succès, ne serait-ce que pour les médecins agréés, dont les indubitables problèmes ne sauraient se résoudre purement et simplement avec la structure tarifaire du TARMED, nous l'avons toujours dit. Dès lors, nous allons développer un «tarif pour médecins agréés». Cela étant, une étape décisive a été franchie par l'accord trouvé sur l'introduction du TARMED dans le domaine de l'AA/AI/AM pour le $1^{\text {er }}$ mai 2003. Les sociétés cantonales peuvent nous être reconnaissantes d'avoir réussi, dans la perspective de la création d'un tarif fondé sur l'économie d'entreprise, à fixer la valeur du point tarifaire à Fr. 1.-.

La $2^{\mathrm{e}}$ révision de la LAMal est à long terme d'une portée beaucoup plus grande, avec la levée de l'obligation de contracter au centre des débats. Après le naufrage du projet au Conseil national, le Conseil des Etats est à nouveau passé à l'offensive en essayant de donner à la loi un caractère d'économie de marché. Nul ne sait encore à quoi ressemblera la loi révisée après les retouches finales. On est en droit de penser que la levée de l'obligation de contracter sera fixée dans une forme modifiée, ce qui relancera définitivement la question d'un référendum par la FMH.

Si la position de la FMH dans les médias, donc dans le public, peut être qualifiée de meilleure que par le passé, elle demeure néanmoins des plus fragiles. Les incessantes querelles portées devant les médias et l'attitude de rejet envers tout changement ont conduit l'un des journaux les plus renommés en Suisse à nous reprocher, une fois de plus, de faire de l'opposition fondamentaliste et sans imagination. Ce n'est pas ainsi que l'on gagnera des voix. Il va falloir que nous fournissions de grands efforts pour présenter nos vues, dans le débat politique, sur l'assistance médicale de la population suisse dans un monde en rapide mutation et prendre au sérieux les critiques qui nous sont adressées. Dans un monde où la crise économique larvée abrite les germes d'une lente et constante dégradation, les attitudes autoritaires de nombre de nos membres, avec leur souhait irrépressible de «taper du poing sur la table» ne peuvent que susciter l'indifférence.

La FMH ne vit plus une ère florissante. Le Comité central et le Secrétariat général sont parvenus dans tous les domaines aux limites de leurs performances. Notre aptitude à assumer de nouvelles tâches, sans ressources supplémentaires, est quasiment épuisée. Il nous faudra limiter notre offre de prestations, ce qui devrait se révéler difficile pour beaucoup de non-membres et d'organisations dans le domaine de la santé publique. 2002 était une année au cours de laquelle nous avons dû faire face aux difficultés avec des ressources de plus en plus serrées.

Ce rapport n'est guère optimiste, je l'avoue. Mais son objectif est aussi de refléter la réalité. Sans aller jusqu'à devoir promettre, comme Churchill en 1940, du sang, de la sueur et des larmes, convenons que nous serons confrontés, les années prochaines, à des défis particulièrement difficiles à relever. Un sens des réalités et une fidélité à une médecine axée sur le patient devraient nous faire balayer ces inquiétudes.

Dr Hans Heinrich Brunner 


\section{Rapports des membres du Comité central}

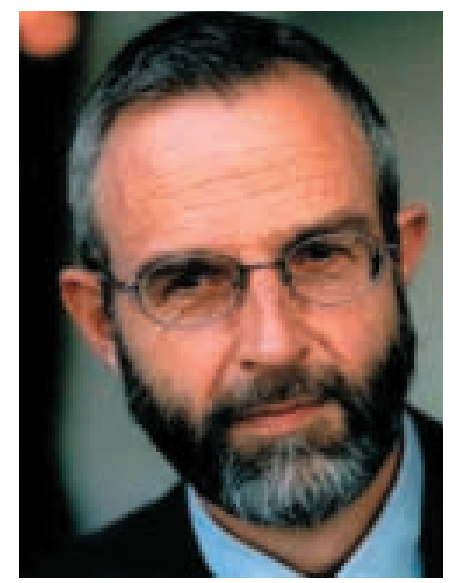

Hans Heinrich Brunner

\author{
1. Dr Hans Heinrich Brunner, membre du CC depuis 1984, \\ président de la FMH depuis 1994, département assurance et promotion \\ de la qualité de la FMH
}

Où voyez-vous aujourd'hui, dans votre domaine d'activité, le plus grand et le plus pressant besoin d'agir?

La sécurité des patients (inhérente à la gestion du risque) reste prioritaire dans le domaine de l'assurance-qualité (ou encouragement à une qualité toujours plus élevée, la qualité étant un processus, non pas un état). A cet effet, nous soutenons activement les médecins dans l'exercice de leur profession, p.ex. avec la mise en place de systèmes informatisés d'annonce d'incidents critiques (CIRSmedical).

Nous avons donc pris contact avec des infirmiers et des médecins de divers domaines spécialisés car il est important de prendre en considération leur savoir-faire ainsi que le mode de fonctionnement des différents établissements hospitaliers en matière de notification d'incidents. De tels systèmes sont déjà opérationnels dans les cabinets de groupe HMO. Dans une première phase, nous voyons l'importance des CIRSmedical avant tout dans la construction d'une «culture professionnelle», dans laquelle la réflexion personnelle et la discussion objective des processus et structures ont leur place sans interférence émotionnelle. Nous le répétons: ces systèmes d'annonce ne remplacent pas la gestion du risque mais en font partie.

\section{Dr Ursula Steiner-König, département de prévention et santé publique, au Comité central depuis 1994, vice-présidente de la FMH depuis 2000}

Quelles ont été vos activités en tant que membre du CC au cours de la période sous revue?

- Présence au sein de plusieurs commissions fédérales dans le domaine de la prévention et de la santé publique:

- Commission fédérale et projet partiel «Fumer, ça fait du mal»: formation continue pour médecin: Association suisse pour la prévention du tabagisme,

- Projet: «Ça débouche sur quoi?», sur les risques de l'alcoolisme,

- Commission fédérale sur les questions concernant le sida,

- Communauté nationale de travail «Politique de la drogue»,

- Fondation pour la promotion de l'allaitement maternel,

- Politique nationale de la santé, axe prioritaire: santé psychique;

- Présence au sein de la Commission centrale d'éthique (CCE) de l'ASSM;

- Direction de la Commission de recours pour les titres de formation postgraduée;

- Consultations:

- Vote sur le régime des délais en ce qui concerne l'interruption de grossesse,

- Loi sur la recherche embryonnaire,

- Projet de fondation d'une organisation faîtière sur le plan suisse pour la prévention du suicide.

Quelles ont été vos tâches les plus importantes?

- Groupe de travail prévention du suicide;

- Loi sur la recherche embryonnaire;

- CCE.

Quels sont les objectifs que vous vous êtes donnés et lesquels avez-vous atteints?

- Apporter une réflexion d'ordre éthique dans tous les domaines qui s'y prêtent, tout particulièrement dans la relation médecin-patient.

- Prendre constamment la défense des médecins praticiens dans les groupements précités.

- Indiquer, en matière de projets de prévention, les divergences entre projets pratiques et théoriques, ainsi que leur application dans la pratique. 


\section{Quel a été votre plus grand succès?}

- Participation à la sous-commission de l'ASSM qui a mis au point la directive sur l'«exercice de la profession médicale auprès de personnes incarcérées».

- Votation sur la solution des délais.

\section{Quelles sont les difficultés rencontrées et votre plus grande contrariété?}

\section{FMH interne}

Manque de temps pour approfondir certaines questions. Le manque de temps pour les contacts avec la responsable de projets du département ou - selon le cas - avec le service juridique de la FMH.

\section{FMH externe}

- Problème de la «psychothérapie déléguée».

- Activités se recoupant et manquant de coordination entre les institutions du domaine de la prévention du tabagisme.

- Manque de courage civique du Conseil fédéral par rapport à l'augmentation de l'impôt sur le tabac. Manière discutable d'aborder la politique de l'asile: les personnes provisoirement admises ont besoin de soutien dans les domaines aussi bien sanitaires que professionnels, de manière à les préparer le mieux possible au retour dans leur pays (qui n'est pas toujours volontaire) et aux difficultés qui les attendent.

\section{Quels sont les événements politiques ou les développements qui ont eu des conséquences} sur votre domaine d'activité?

- Entrée en vigueur des accords bilatéraux par rapport aux recours en matière de demandes de titres.

- Décision du Conseil fédéral concernant l'impôt sur le tabac.

- Délai concernant la votation sur la dépénalisation de l'interruption de grossesse.

- Remaniement et poursuite des travaux du programme national sur le VIH-sida.

- Révision de la loi sur les stupéfiants.

\section{Dans quel domaine d'activité voyez-vous aujourd'hui le plus grand et le plus pressant} besoin d'agir?

- Politique nationale de la santé, objectif principal: santé psychique. Indépendamment de cet objectif tout en y étant lié: projet de l'organisation faitière de prévention du suicide. Comment créer d'utiles interdépendances.

- Révision de la loi sur les stupéfiants.

- Travaux de la CCE de l'ASSM.

- Poursuite de projets de prévention dans le domaine du tabagisme et de l'alcoolisme.

\section{Quel message souhaitez-vous adresser}

\section{aux membres de la FMH}

Tout en comprenant l'importance de la question des revenus individuels, les membres de la FMH ne devraient pas oublier qu'en dépit de toutes les divergences qui certes, sont réelles, la confiance et la solidarité sont nécessaires afin de permettre au CC de représenter tous les membres de la FMH au mieux de ses possibilités et d'y parvenir, je l'espère. Je saurais également gré aux médecins en cabinet médical de ne pas oublier la relève.

au président de la FMH

Il est parfois difficile de comprendre les raisons de ta discrétion dans certains domaines, mais tu mérites toute notre reconnaissance pour ton engagement sans faille.

\section{à M. Pascal Couchepin}

L'être humain ne saurait se réduire à une équation prévisible. Il serait vain de vouloir manipuler des malades psychiques comme des pions. Il en va de même des demandeurs d'asile, eux aussi des êtres humains. Parce que ces derniers, précisément, doivent quitter notre pays au bout d'un certain temps, nous devons les y préparer le mieux possible afin qu'ils puissent maîtriser les difficultés qui les attendent dans leur pays d'origine dans un état de santé le meilleur possible. 


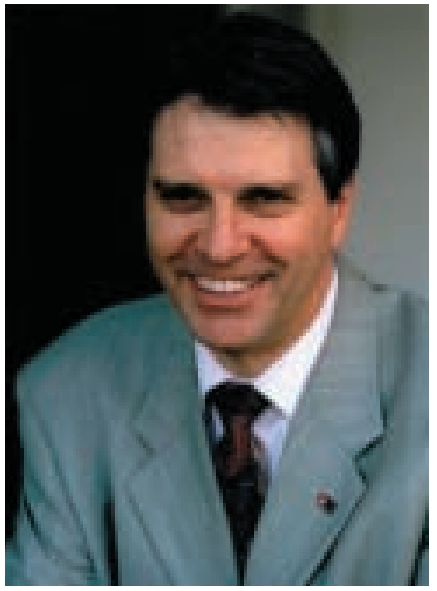

Yves Guisan

\section{Dr Yves Guisan, membre du Comité central depuis 1987, vice-président de la FMH depuis 1997}

Indépendamment de mon activité parlementaire consacrée à la politique sanitaire dans son ensemble, 2 volets principaux ont retenu mon attention: la médecine hospitalière avec $\mathrm{H}+$, ainsi que la Commission fédérale de statistiques sanitaires. Parallèlement j'ai exercé une vaste activité de relations publiques pour le comité central, que ce soit auprès des médias, de différentes sociétés médicales ou autres associations sur mandat du président ou à la suite de leur sollicitation directe.

H+ s'est trouvée confrontée, tout comme la FMH, avec la mise en place du TARMED. Même si la direction générale du projet regroupe les différents partenaires, il était important que les médecins hospitaliers puissent exprimer leur point de vue au sein de l'association des hôpitaux de Suisse, dont ils sont un élément déterminant du fonctionnement. De plus H+ a entrepris un gros effort de réforme de ses structures et de positionnement sur la scène fédérale du système de santé. Les médecins hospitaliers ne sauraient y être indifférents.

La statistique sanitaire est indiscutablement un élément clé du pilotage du système de santé. Elle souffre de lacunes majeures reconnues de toute part. Curieusement cette situation se heurte à l'absence de toute volonté politique. La situation déplorable des finances fédérales ne laisse guère entrevoir d'amélioration. Dans ces conditions, la navigation à vue ne peut malheureusement que rester la règle, malgré les appels du pied de la Commission fédérale de statistiques sanitaires qui n'a quasiment plus que cette seule mission.

Le bilan de l'année 2002 est plutôt mitigé. La FMH se trouve constamment prise entre deux feux: celui de ses membres qui ont une vision trop unilatérale des dossiers avec la conviction qu'il suffit de taper sur la table pour avoir raison; et celui des milieux politiques et des assureurs convaincus de résoudre définitivement tous les problèmes du système de santé au moyen de quelques raccourcis déconcertants comme la suppression de l'obligation de contracter, la clause du besoin, ou le financement moniste des hôpitaux. Comment dès lors ouvrir encore une voie raisonnable susceptible de trouver grâce auprès des uns et les autres?

Il importe surtout dans ces conditions de dégager un message clair: d'un côté, les médecins sont prêts à assumer leurs responsabilités en matière d'assurance sociale et de santé publique. Mais cela ne peut se faire que par le biais d'accords contractuels négociés dans un climat de confiance et de respect de l'intérêt général. Aucune solution ne verra jamais le jour dans l'atmosphère de suspicion, de non-dits, et surtout de répression larvée qui règne actuellement. Une politique constructive ne peut s'établir qu'en fonction des objectifs poursuivis, libre accès au traitement, solidarité, qualité, efficacité, économicité du traitement, et non avec la seule intention de lutter contre les abus de quelques moutons noirs!

\section{Dr Max Giger, membre du Comité central depuis 1987, département «medical education» et département médicaments}

Quelles ont été vos activités en tant que membre du CC au cours de la période sous revue?

Direction du département de la formation médicale

- Application de la RFP et de la RFC;

- Collaboration avec les facultés de médecine;

- Présidence

- de la Commission pour la formation postgraduée et continue (CFPC),

- Commission des titres,

- Commission des établissements de formation postgraduée;

- Représentation de la FMH dans les groupements suivants:

- Commission interfacultés médicale suisse (CIMS),

- Union européenne des médecins spécialistes (UEMS).

Direction du département des médicaments

- Représentation du corps médical dans les questions d'application des directives juridiques en matière de médicaments;

- Représentation du corps médical dans la Commission fédérale des médicaments (CFM);

- Présidence du Conseil de fondation pour la sécurité des médicaments (SAS/CHDM). 
Membre des comités d'administration et de surveillance suivants:

- FMH Services;

- Fondation de prévoyance du personnel PAT;

- Caisse de compensation de l'AVS medisuisse.

Quelles ont été vos tâches les plus importantes?

- Application de la RFP et de la RFC;

- Coopération avec les facultés de médecine;

- CFM.

Quels sont les objectifs que vous vous êtes donnés et lesquels avez-vous atteints?

Assurance-qualité de la formation postgraduée médicale par des enquêtes sur l'appréciation de la qualité de la formation postgraduée par les médecins assistants, par des concepts écrits et leur évaluation dans le cadre de visites.

- La $6^{e}$ enquête a été menée avec succès. Le projet de nouveau questionnaire est en cours d'exécution. Une grande partie des établissements de formation a renvoyé les concepts de formation dans les délais prescrits.

- Les manuels en tant que base des visites d'établissements sont à la disposition de toutes les sociétés de discipline. Les premières visites ont eu lieu avec trois experts, l'un représentant la société de discipline, l'autre la CFPC et le troisième l'ASMAC.

Développement de la collaboration avec les facultés de médecine, en particulier dans le domaine de la formation postgraduée médicale.

- Les questions sur l'enseignement, la formation postgraduée, la recherche et l'avenir de la profession de médecin en Suisse ont été discutées au sein de groupes de travail sur des thèmes précis.

Meilleure application des critères d'efficacité, d'adéquation et d'économicité (EAE) concernant l'admission de médicaments dans la liste des spécialités et la rationalisation du travail en tant que membre de la CFM.

- Les annonces de nouveaux médicaments devront, à l'avenir, se faire au travers d'un document de synthèse contenant les données scientifiques sur l'efficacité et l'économicité en matière de pharmacologie.

\section{Quel a été votre plus grand succès?}

Accord donné par la CFPC pour la création d'une attestation (depuis longtemps souhaitée) de formation complémentaire (AFC) en tant que médecin-conseil.

\section{Quelles sont les difficultés rencontrées et votre plus grande contrariété?}

\section{FMH interne}

- Manque de sens des réalités de la part de nombreux responsables de la formation postgraduée par rapport aux mesures d'assurance-qualité prises par les médecins eux-mêmes et des travaux non négligeables entrepris à cet égard qui sous-tendent l'ensemble du processus de la formation postgraduée médicale.

- Lourdeur des organes de formation postgraduée par rapport à la réaction sur les propositions de réforme, telles que la structure modulaire des programmes de formation postgraduée, la répartition des établissements de formation orientée sur des processus ou l'introduction d'un module de management dans les programmes de formation.

FMH externe

- Blocage de l'accès à la pratique privée, qui n'a fait que conduire à un afflux préventif massif de demandes de titres, la plupart du temps pour le titre de formation postgrade de «médecin praticien».

- Pression mise sur la formation postgraduée en raison de temps de travail raccourcis, adaptés aux prestations. Intentions exprimées par les milieux politiques du social et de la formation, par des économistes de la santé et des fonctionnaires des caisses-maladie de reporter sur le corps médical les coûts de la formation postgraduée médicale qui jusqu'ici, étaient à la charge de la société (comme dans tous les pays européens), au sens d'un investissement permettant un bon fonctionnement du système de santé. 
Quels sont les événements politiques ou les développements qui ont eu des conséquences sur votre domaine d'activité?

- Le blocage de l'accès à la pratique privée a conduit à un afflux aberrant de demandes de titres.

- A la suite des débats sur le TARMED, nombre de questions de formation postgraduée sont abordées sur le plan des prestations et de leur rémunération, occultant ainsi le réel objectif qui doit être d'optimiser la formation postgraduée pour servir l'activité médicale et le bien du patient.

Dans quel domaine d'activité voyez-vous aujourd'hui le plus grand et le plus pressant besoin d'agir?

Les programmes de formation postgraduée doivent être révisés avec pour objectif d'obtenir une formation plus efficace, modulée sur les besoins spécifiques de la discipline et tenant compte de la mutation des structures sociales, de la réduction du temps de travail normal et de la féminisation de la médecine. Un catalogue des objectifs de formation doit être mis au point pour chaque discipline. Les méthodes modernes d'apprentissage, telles que l'enseignement mixte et exhaustif par voie électronique (blended and enhanced e-learning) doivent être introduites dans le but d'augmenter l'efficacité.

Les frais d'une future formation postgraduée médicale plus efficace doivent être explicitement pris en charge par les organes politiques concernés si l'on veut maintenir l'assistance médicale actuelle de la population.

\section{Quel message souhaitez-vous adresser}

aux membres de la FMH

Le soutien à des projets de formation postgraduée et continue efficaces et axés sur le patient permet à chacun de nous de progresser.

au président de la FMH

Que ta lutte continue, pour la FMH et un corps médical toujours meilleur!

\section{à M. Pascal Couchepin}

Nous avons besoin d'un appareil législatif léger, à même de garantir un art médical axé sur le patient et de déléguer au corps médical la responsabilité et la compétence de son exécution.

Une application plus stricte des critères EAE dans le domaine des médicaments et une rationalisation de la liste des spécialités qui l'accompagnerait pourrait faire gagner en efficacité.

La législation sur les médicaments devrait être adaptée au quotidien clinique, impliquant une approche prospective des effets indésirables des médicaments dans un système de déclaration Sentinella.

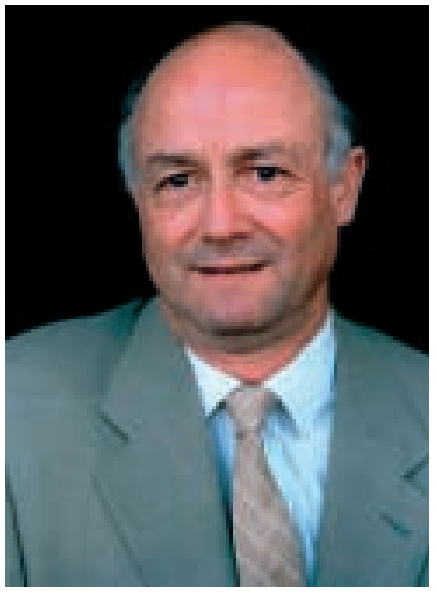

Claude Aubert

\section{Dr Claude Aubert, membre du Comité central à partir de 1994}

Les activités en tant que membre du Comité central pendant la période sous revue A participé à la Commission centrale d'éthique de l'Académie suisse des sciences médicales (CCE / ZEK) et aux séances de la Commission interfacultaire médicale suisse (CIMS).

\section{Les charges les plus importantes}

A suivi l'actualité politique et rédigé des éditoriaux.

A informé et cherché à mobiliser les lecteurs du BMS sur le plan de la politique professionnelle.

\section{L'impact}

L'écriture!

\section{Les difficultés rencontrées}

- Se plaint du cloisonnement et de la méfiance.

- Dénonce la courte vue.

- S'achoppe à l'incohérence des débats en matière de santé publique. 
Les défis

Recherchera des idées politiques utiles aux médecins.

Aimerait dire

aux membres de la FMH:

Militez!

au président de la FMH:

Cap sur l'avenir de la profession !

à Pascal Couchepin

Bon vent!

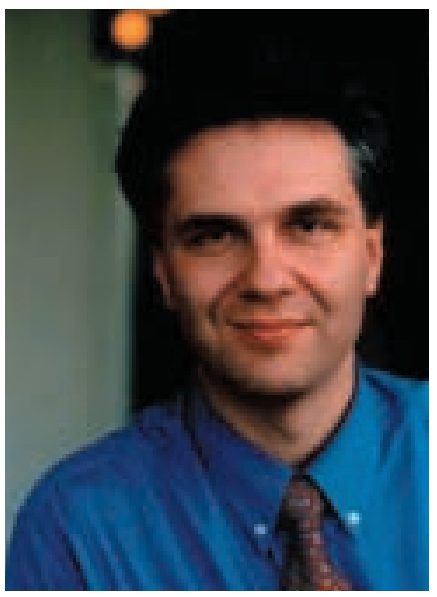

Ludwig T. Heuss

\section{Dr Ludwig T. Heuss, membre du Comité central depuis 1994; domaine «médecins et profession médicale»}

Quelles ont été vos activités en tant que membre du CC au cours de la période sous revue?

- Tout d'abord au sein du CC lui-même, avec des ordres du jour chargés, des discussions conceptuelles, des séances en conclave, des travaux inhérents au domaine dont je suis chargé, ainsi que des travaux à la maison;

- Président de la Commission de recours pour les établissements de formation postgraduée nouvellement créée;

- Membre du comité des Editions médicales suisses EMH SA;

- Membre du comité de la Caisse de compensation des médecins medisuisse AVS AI;

- Membre du comité de la caisse de secours des médecins suisses;

- Interlocuteur de la société de médecine du canton de Bâle et de l'ASMAC.

\section{Quelles ont été vos tâches les plus importantes?}

- Le domaine «Médecins et profession médicale» a principalement été confronté à des questions concernant les perspectives de développement à long terme pour la nouvelle génération de médecins, notamment le blocage absurde et dirigiste de l'accès à la pratique privée (cf. rapport de gestion).

- Une association professionnelle forte a également besoin d'un organe de publication médicoscientifique de haut niveau. L'extension et le renforcement des Editions médicales EMH font partie de cet objectif.

- Les organes décisionnels courent facilement le risque de perdre le contact avec la base. De bons rapports et une fonction de lien avec une société cantonale de médecine et avec le président de l'ASMAC ont été très importants pour moi.

Quels sont les objectifs que vous vous êtes donnés et lesquels avez-vous atteints?

En 1997, nous avons pour la première fois et malgré de fortes résistances, discuté de propositions concrètes concernant la question difficile du remaniement structurel des prestations de formation postgraduée dans nos hôpitaux. Dans l'intervalle, l'idée d'une fonction de médecins spécialistes hospitaliers a fait son chemin dans le monde médical et politique et différents modèles sont en cours d'élaboration. Cette évolution est réjouissante, même si les répercussions liées au blocage de l'accès à la pratique privée n'ont rien de positif et que certains aspects se révèlent contraires à l'idée initiale. Je maintiens cependant l'idée que nous devons créer de nouveaux postes et modèles d'activité, faire preuve d'imagination, de flexibilité et être prêts à prendre des décisions, afin de satisfaire aux nouvelles exigences de la médecine. J'aimerais en outre inciter les gens à développer de nouvelles idées parallèlement aux profils professionnels traditionnels.

Quel a été votre plus grand succès?

Il n'y pas eu de grands succès, mais plutôt des petits succès personnels, par exemple amener de bons arguments dans un débat et réussir à persuader, avoir obtenu la possibilité de décider lors d'une votation ou peut-être, dans un débat avec Otto Piller, être arrivé à ce qu'il soit à court d'arguments. 


\section{Quelles sont les difficultés rencontrées et votre plus grande contrariété?}

\section{FMH interne}

Il règne au sein du corps médical une forte solidarité et ce, malgré les divergences et les différences personnelles. Le résultat de la votation générale sur le TARMED et les réactions sur le blocage de l'accès à la pratique privée l'ont bien montré. Cela me contrarie cependant que de nombreux membres ne voient en la FMH qu'un organe de contrôle et de surveillance. En tant que membres du CC, nous devons donc travailler sur notre image et mieux informer de l'importance des décisions à prendre, notamment lorsqu'elles comportent des restrictions.

\section{FMH externe}

Tout le monde s'acharne sur les médecins, mais lors des débats politiques il n'y a plus réellement d'adversaires. Les politiciens ne disent pas à leurs électeurs les choses telles qu'elles sont et les caissesmaladie ne sont, de par leur nombre, pas des partenaires fiables.

\section{Quels sont les événements politiques ou les développements qui ont eu des conséquences} sur votre domaine d'activité?

Le blocage inattendu et injustifié de l'accès à la pratique privée en tant que mesure d'urgence en juin dernier, ainsi que la suppression de l'obligation de contracter qui s'annonce.

\section{Dans quel domaine d'activité voyez-vous aujourd'hui le plus grand et le plus pressant besoin d'agir?}

En Suisse, nous sommes confrontés à d'importantes difficultés de répartition des soins médicaux. Cela concerne tout particulièrement le besoin des hôpitaux en postes transitoires et l'assistance médicale dans certaines régions. Le marché libre n'a pas permis de résoudre ces problèmes et il est tout aussi absurde de croire que les mesures d'urgence précitées permettront d'aplanir ces inégalités. Selon moi, le plus pressant besoin d'agir concerne la structure organisationnelle des hôpitaux, qui doivent réviser leur point de vue selon lequel leur activité-clé (les prestations médicales) peut être effectuée principalement par des médecins dont les $80 \%$ occupent une fonction transitoire.

\section{Quel message souhaitez-vous adresser}

aux membres de la FMH

Aidez-nous à rendre le corps médical fort sur le plan de la politique de la santé et à maintenir le libre exercice de la profession axé sur les besoins des patients et les progrès scientifiques.

au président de la FMH

Qu'il pense également à sa propre santé!

\section{à M. Pascal Couchepin}

Une libre concurrence entre l'offre et la demande se fondant sur un marché de la santé libéralisé ne signifie pas uniquement réglementer les prix et les quantités, mais entraîne aussi l'exclusion de groupes importants de la population (cf. Etats-Unis). On ne peut pas uniquement se référer aux côtés positifs de la libre concurrence et omettre les aspects négatifs. 


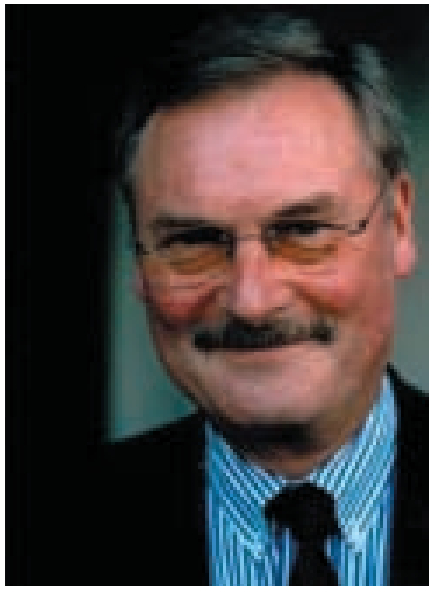

Reto Laetsch

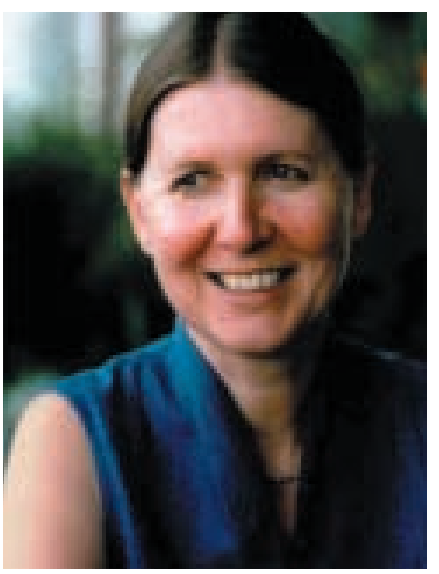

Marie-Christine Peter

\section{Dr Reto Laetsch, au Comité central depuis 1994}

Quelles ont été vos activités en tant que membre du CC au cours de la période sous revue?

Médecine d'urgence et de sauvetage

- Programme de la FMH pour le domaine du sauvetage en Suisse;

- Responsabilité au sein de la Swiss Medical Rescue and Emergency Conférence

(Commission médicale suisse de sauvetage [SMEDREC]);

- Forum «sauvetage en Suisse » de la FMH;

- Comité du service sanitaire coordonné de l'armée (SSC).

Délégation aux négociations sur la rémunération des médicaments basée sur les prestations (RBP). Commission fédérale des prestations (CFP).

Union européenne des médecins omnipraticiens (UEMO).

Commission du compendium.

\section{Quelles ont été vos tâches les plus importantes?}

Un domaine important a été constitué par les négociations sur la RBP. L'objectif était d'élaborer avec santésuisse, d'ici à l'adoption de la valeur du point et à la fin des négociations sur le TARMED, un modèle de distribution et un modèle de contrat à l'échelle cantonale. Avant la fin de ces travaux et l'obtention des résultats destinés aux instances décisionnelles de la FMH, quelques obstacles doivent encore être franchis.

Un autre domaine important a été celui du sauvetage. Avec les organisations faîtières, l'Interassociation de sauvetage (IAS) et la Croix-Rouge suisse (CRS), ainsi qu'avec d'autres partenaires, notamment le Département fédéral de la défense, de la protection de la population et des sports (DDPS) et l'Association suisse des ambulanciers professionnels (ASA), il s'agissait de répartir les tâches entre les diverses organisations et de focaliser les activités dans le domaine du sauvetage. Dans le but d'assurer cette bonne coordination à l'avenir, un forum sur le sauvetage aura lieu tous les deux ans, réunissant toutes les organisations actives dans ce domaine.

Le programme de la FMH pour le domaine du sauvetage en Suisse définit la liste des éléments d'équipement des médecins de service. Elle a été publiée sur le site internet de la FMH. Les travaux de «Catégorisation des unités d'urgence» ont été développés, sur le plan de la conception, de manière à intégrer avec succès le projet «Information et engagement dans le service sanitaire» du SSC. L'objectif qui demeure à réaliser est celui de la préparation du thème «psychiatrie en médecine d'urgence» en vue d'en débattre dans le cadre de la formation prégraduée, postgraduée et continue des médecins de service et d'urgence.

\section{Dr Marie-Christine Peter, au Comité central depuis 1997}

Quelles ont été vos activités en tant que membre du CC au cours de la période sous revue? J'ai siégé au sein de diverses commissions et organisations:

- Commission fédérale des prestations (CFP): consultation du DFI concernant l'adoption de prestations controversées dans le catalogue des prestations et, tout particulièrement, appréciation des prestations sur la base de critères d'efficacité, d'adéquation et d'économicité (EAE). Se fondant sur le changement à la direction du DFI, la FMH espère que l'on fasse le bilan des tâches et du mode de travail de la commission au cours de l'année. Sur mandat de la CFP, j'ai coopéré au groupe de travail «Garantie de prise en charge» et «Dépistage par mammographie».

- Pour la délégation aux négociations des sociétés cantonales avec santésuisse (G7), l'année 2002 a été une année riche de travail. En tant que déléguée du Comité central, avec statut d'observatrice, j'ai pris part à diverses séances, notamment au sujet de la convention-cadre signée le 5 juin 2002 par la FMH et santésuisse. Depuis, il a fallu mettre au point les diverses annexes.

- Oncosuisse: le forum national de tous les protagonistes œuvrant dans le domaine du cancer a reçu un mandat de la CDS et de l'OFSP pour la mise sur pied d'un programme national en la matière. Les tâches de la FMH ne sont pas encore clairement définies.

- SMSR: les contacts réguliers entretenus avec la Société médicale de la Suisse Romande ont permis une meilleure représentation au sein du CC des enjeux spécifiques à la Suisse romande. 


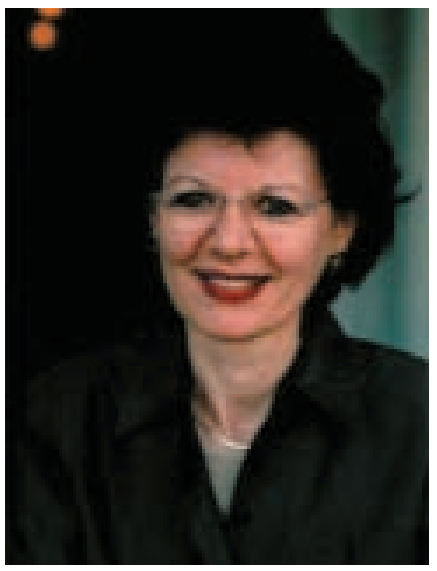

Verena Briner
- Contacts avec la base: séance en conclave de la Société suisse de médecine interne (SSMI), de la Société suisse d'allergologie et d'immunologie clinique (SSAIC) et de la Société médicale du Valais.

\section{Pr Verena Briner, au Comité central depuis 2000}

Quelles ont été vos activités en tant que membre du CC au cours de la période sous revue? Je représente la FMH au sein de la Commission d'experts pour le secret professionnel dans la recherche médicale. Celle-ci traite régulièrement des demandes concernant la levée du secret médical à des fins de recherche dans le domaine de la médecine et de la santé publique. Ce travail s'effectue lors de séances à Berne ou par circulaires électroniques.

Je représente la FMH au sein de la commission de la Liste des moyens et appareils (LIMA). Des demandes y sont traitées, émanant généralement de firmes souhaitant l'intégration de moyens destinés au domaine ambulatoire (p. ex. lingerie pour incontinence, pompes à lait maternel, matériel de pansements, etc.), en collaboration avec des représentants de divers milieux (patients, firmes, caisses, Swissmedic).

Quels sont les objectifs que vous vous êtes donnés et lesquels avez-vous atteints?

Traitement efficace et sérieux des demandes. Représentation des intérêts des médecins praticiens. Pour une part, ces objectifs ont été atteints.

\section{Quel a été votre plus grand succès?}

Pas de grand succès à déclarer.

Quelles sont les difficultés rencontrées et votre plus grande contrariété?

\section{FMH interne}

Aucune.

\section{FMH externe}

Une difficulté a été de montrer que la recherche est indispensable si l'on veut des progrès. Nous profitons d'études et de résultats d'années précédentes et devrions dès lors accepter l'utilisation de ces données au profit de la science. Il en va de même du matériel d'examen (sang, biopsies, etc.). Il convient de relever qu'actuellement, le droit de l'individu prime sur le droit de la communauté, laquelle en fait subventionne la médecine alors que le patient n'a pas à s'acquitter du coût entier des bienfaits qu'il en retire. Il n'y a plus que des droits et pas de devoirs.

Quels sont les événements politiques ou les développements qui ont eu des conséquences sur votre domaine d'activité?

Aucun.

Dans quel domaine d'activité voyez-vous aujourd'hui le plus grand et le plus pressant besoin d'agir?

Je l'ai déjà évoqué plus haut. L’hostilité envers la recherche scientifique est dramatique.

\section{Quel message souhaitez-vous adresser}

aux membres de la FMH

Motiver la population et en appeler à sa responsabilité pour contribuer au progrès de notre médecine en mettant à disposition leurs données et résidus de matériaux testés pour des objectifs de recherche. 


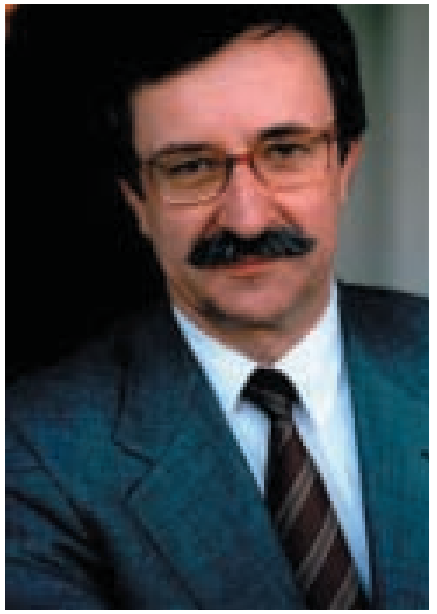

Franco Muggli

\section{Dr Franco Muggli, membre du Comité central depuis 2000}

Quelles ont été vos activités en tant que membre du CC au cours de la période sous revue? Le temps court, 12 mois d'activité peuvent paraître très longs et pourtant c'est uniquement à la fin de l'année que l'on se rend compte de ce que l'on aurait voulu faire et de ce qu'il reste à faire. En bref, l'activité au sein du Comité central a demandé la patience et la volonté de partager et de soutenir la stratégie et la tactique du président dans des moments extrêmement difficiles en matière de politique professionnelle. C'est d'ailleurs ce que l'on attend des membres d'un «conseil», à savoir réfléchir sur les stratégies et s'exprimer quant aux tactiques proposées. En outre, on demande aussi à ces mêmes membres d'être unis et loyaux dans l'exercice de leur fonction de conseiller, même s'il faut parfois faire le poing dans sa poche.

\section{Quelles ont été vos tâches les plus importantes?}

La tâche qui m'a demandé le plus d'engagement au cours de la période sous revue (et aujourd'hui encore) fut la participation aux groupes de délégation avec santésuisse. Il s'agit notamment du G7 sur le plan national, ainsi que l'activité de consultant explicitement exigée par l'Ordine dei medici sur le plan cantonal. Pourquoi cette double fonction? Pour la simple et bonne raison qu'il est important de représenter au sein du G7 tant le Comité central que la société cantonale de médecine italophone. Cela permet ainsi de ne pas perdre le savoir-faire acquis au cours de ces quelque 6 années d'activité politico-professionnelle.

Une autre tâche également très importante a consisté à publier la version italienne du TARMED, tâche complexe et de longue haleine qui a demandé énormément de temps et de nombreuses vérifications. En fait, la traduction en italien a souvent été compromise par le souci de vouloir rendre le plus fidèlement possible les expressions et les termes issus de l'allemand fédéral ... Nous espérons cependant que les amoureux de la langue de Dante ne nous en tiendront pas rigueur!

Une troisième activité qui mérite d'être mentionnée a concerné une collaboration plus concrète avec le Service médical et sanitaire de l'armée, notamment notre présence au sein de la direction du groupe de projet pour la création d'une Académie de médecine militaire. Ces dernières années, on a assisté à une diminution progressive du nombre de jeunes médecins diplômés disposés à poursuivre leur carrière militaire. Grâce au projet d'Académie de médecine militaire et aux accords passés entre la FMH et le sous-groupe sanitaire, on cherche à rendre cette activité plus attrayante (également sur le plan professionnel). On constate que les choses évoluent dans ce sens, le recrutement dans le cadre de la réforme Armée XXI en est un exemple.

Quels sont les objectifs que vous vous êtes donnés et lesquels avez-vous atteints?

Les objectifs résultant des 3 activités précitées ont été atteints. Il s'agit cependant d'un travail de longue haleine avec de bonnes chances d'obtenir les résultats escomptés.

\section{Quel a été votre plus grand succès?}

Avoir réussi à ce que mes patients gardent confiance en leur médecin de premier recours! En effet, on peut aisément imaginer combien il est difficile de gérer son propre cabinet de médecine générale, et non une discipline plus spécifique, tout en s'engageant dans la politique professionnelle, dans le cadre des tarifs et de la formation postgraduée médicale.

\section{Quelles sont les difficultés rencontrées et votre plus grande contrariété?}

Eh bien, lorsque mes interlocuteurs se mettent spontanément à échanger leurs pensées dans un sabir issu d'une vallée alpine des plus reculées, alors il est difficile de ne pas se sentir quelque peu découragé. Je crois cependant plus en la recherche de solutions qu'en l'énumération de difficultés. Quoi qu'il en soit, ce ne fut guère agréable de lire les statistiques du Dr Halser dans le Bulletin des médecins suisse sans en avoir préalablement été informé.

\section{Quels sont les événements politiques ou les développements qui ont eu des conséquences} sur votre domaine d'activité?

Le scénario LAMal 2004 sera déterminant pour l'avenir du libre exercice de la médecine. Nous sommes en présence d'une inconnue car la suppression de l'obligation pour les assureurs-maladie de contracter avec chaque médecin et le fait de déléguer aux cantons le soin de déterminer le nombre de médecins nécessaires (et lesquels) pour garantir les soins ne laissera guère d'espoir à un libre exercice de la médecine. 

œuvre les concepts élaborés!

\section{Quel message souhaitez-vous adresser}

Aux membres de la Fédération:

Ne tirez pas sur le pianiste.

Au président de la FMH:

Le pape n'est pas un mauvais exemple à suivre (les visites papales favorisent beaucoup le consensus).

\section{A Pascal Couchepin:}

Bien que les médecins ne soient pas des producteurs de lait ou de vénérables agriculteurs, ils ont eux aussi une mission importante à accomplir en faveur des citoyens. En effet, pour bien faire les choses, il convient d'être motivé et content de les faire. Une phrase à méditer, Monsieur le Conseiller fédéral!

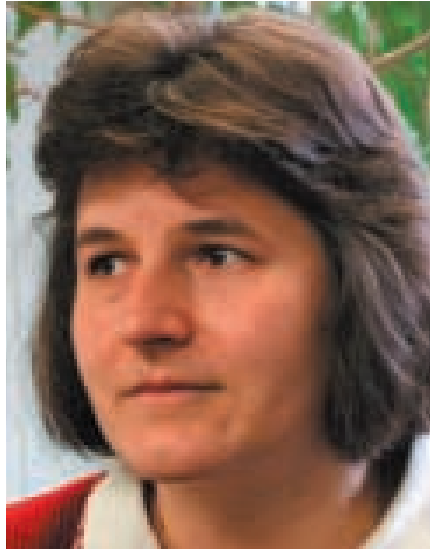

Susanna Stöhr

\section{Dr Susanna Stöhr, au Comité central depuis 2000}

Quelles ont été vos activités en tant que membre du CC au cours de la période sous revue?

- Collaboration aux activités du domaine «Formation médicale», précédemment nommé «Formation prégraduée, postgraduée et continue»;

- Collaboration à des projets dans le domaine de la formation postgraduée;

- Membre de la Commission de recours pour les titres de formation postgraduée (CT TFP);

- Représentation des intérêts des jeunes médecins au sein du CC.

Quelles ont été vos tâches les plus importantes?

- Soutien du responsable du domaine «Formation médicale» et collaboration à tous les projets du domaine de la formation postgraduée;

- Examens de spécialiste;

- Adaptation des programmes de formation postgraduée avant leur mise en vigueur définitive par le CC.

Quels sont les objectifs que vous vous êtes donnés et lesquels avez-vous atteint?

J'ai œuvré pour que l'on fixe les exigences de la formation postgraduée selon des critères d'adéquation et de qualité, et non pas en fonction de dispositions réglementant le nombre de spécialistes. J'ai aussi tenté d'obtenir que les médecins en formation postgraduée soient impliqués dès le départ dans les questions touchant à leur formation.

Plus généralement, il me semble important que l'on garde sans cesse à l'esprit les médecins de la nouvelle génération. Ils représentent un élément important de la FMH et ce sont eux, en fin de compte, qui seront directement touchés par les décisions que nous prenons aujourd'hui.

\section{Quel a été votre plus grand succès?}

Difficile à dire, car c'est souvent une série de petits succès qui vous rapprochent du but. En outre, un succès n'est généralement pas l'œuvre d'une seule personne, mais de plusieurs qui poursuivent ensemble un objectif. N'oublions pas que l'activité au sein du CC est aussi un travail d'équipe.

\section{Quelles sont les difficultés rencontrées et votre plus grande contrariété?}

\section{FMH interne}

Aucune.

\section{FMH externe}

En tant que représentante des jeunes médecins, sans aucun doute le blocage de l'accès à la pratique privée: une décision prise à la va-vite, unilatéralement et sans tenir compte de la qualité, injuste aussi puisqu'elle n'affecte qu'une seule catégorie de médecins, et de plus une décision qui ne fera pas baisser les coûts, bien au contraire. 
Et puis, le fait que l'on ne rate pas une occasion de clouer le corps médical au pilori et de le rendre responsable de tous les maux de la santé publique. Je suis aussi préoccupée par les débats sur la levée de l'obligation de contracter, car les personnes qui entendent prendre les décisions, même si elles sont qualifiées dans leur domaine et font du bon travail, ne sont tout simplement pas à même de juger de la qualité des prestations médicales.

Quels sont les événements politiques ou les développements qui ont eu des conséquences sur votre domaine d'activités?

L'entrée en vigueur des accords bilatéraux et le blocage de l'accès à la pratique privée.

\section{Dans quel domaine d'activité voyez-vous aujourd'hui le plus grand et le plus pressant besoin d'agir?}

Il est essentiel que les programmes de formation postgraduée tiennent leurs promesses. En d'autres termes, les contenus doivent en être modernes et aisément applicables. Il importe aussi que la formation postgraduée puisse être terminée dans les délais prévus. Un titre de spécialiste devrait habiliter son détenteur à assurer de manière autonome des prestations de haute qualité, sans qu'il faille le compléter par d'innombrables attestations de formation complémentaire ou certificats d'aptitude technique.

Pour que tout cela puisse être réalisé, il faut axer la formation postgraduée sur les besoins et prendre des mesures d'assurance-qualité. Dans ce contexte, les postes supplémentaires à créer en vue de la réduction du temps de travail devraient l'être non pas pour des médecins en formation postgraduée mais, dans une mesure adéquate, pour des médecins spécialistes hospitaliers.

La formation postgraduée étant aussi une préoccupation majeure de l'ASMAC, il convient d'impliquer celle-ci dès le début dans les processus concernés.

La formation postgraduée est aussi prodiguée dans l'intérêt des patients et des directeurs des affaires sanitaires. Seuls des médecins bien formés peuvent assurer, maintenant et à l'avenir, une assistance médicale de haute qualité pour la population suisse. Il n'est donc pas question que le financement de la formation postgraduée devienne l'affaire du seul corps médical. Il s'agit de persuader les organes compétents de l'importance de cet enjeu.

\section{Quel message souhaitez-vous adresser}

aux membres de la FMH:

Avec toutes les spécialités qu'elle représente, la FMH est composée de façon très hétérogène. Il est donc inévitable que divers intérêts y cohabitent et que ceux-ci ne puissent pas toujours être défendus à la satisfaction générale. Mais comme les décisions que nous devons prendre concernent l'ensemble des médecins, chacun devrait tenter de réfréner quelque peu ses intérêts particuliers. Ce n'est qu'en donnant une image d'unité que le corps médical parviendra à résister aux vents de plus en plus rudes qu'il doit affronter.

\section{au président de la FMH}

Un vif merci pour son dévouement infatigable au service de notre cause. Cet engagement demande beaucoup de temps, de ténacité et s'apparente souvent à un exercice de haute voltige. Il est évident que personne ne peut faire plaisir à tout le monde, pas même M. Brunner qui n'est pourtant pas loin, dans bien des cas, de réussir la quadrature du cercle ...

\section{à M. Pascal Couchepin}

En prenant des décisions ou en édictant des lois en matière de santé publique, ne voyez pas seulement les coûts et ceux qui en sont prétendument la cause, mais songez aussi au bien des patients. Notre société a besoin d'un système de santé axé sur le patient et fournissant des prestations optimales en fonction de chaque cas particulier. Poursuivre un tel objectif a son prix, et les solutions hâtives ne permettent guère de s'en rapprocher. 


\section{Relations internationales}

\section{Comité Permanent des Médecins Européens (CPME) / Conférence Européenne des Ordres des Médecins (CEOM, anciennement Conférence Internationale des Ordres C.I.O.)}

La Suisse doit-elle ou non demander son adhésion à ces organisations en tant que membre ordinaire? La question est devenue d'actualité depuis la conclusion des accords bilatéraux. Les statuts de ces deux organisations ne permettent toutefois pas à des associations sises dans un Etat tiers d'y adhérer et, dans la perspective proche de l'élargissement de l'UE aux pays de l'Est, leur modification n'entre pas en ligne de compte pour l'instant. La Suisse devra donc continuer à se contenter d'y occuper un strapontin d'observateur. Néanmoins, vu la masse de travail qui incombe souvent à ces organisations, le statut actuel ne comporte pas que des inconvénients.

Alors que la CEOM se consacre principalement aux questions de politique professionnelle, le CPME exerce plutôt une fonction de coordinateur dans le concert des organismes européens (CEOM, UEMO, UEMS, AEMH, etc.). Tous les Etats ne sont pas, comme la Suisse, représentés dans ces deux organes par une seule et même association médicale. Et tous ne partagent pas non plus les vues du président actuel du CPME, l'Autrichien Rainer Brettenthaler, qui cherche à renforcer la coopération entre les diverses associations dans un processus de concentration.

Cela dit, l'Europe se débat dans les mêmes problèmes que les nôtres: la reconnaissance mutuelle des titres de spécialiste, de loin pas résolue, le libre exercice des professions médicales, qui soulève une foule de questions; les horaires de travail excessifs, le sponsoring grandissant de l'industrie et autres maux qui rendent le quotidien difficile. Sans parler des problèmes éthiques qui se posent avec les progrès de la médecine et le fossé qui va s'élargissant dans le domaine de la santé entre l'Est et l'Ouest.

Annamaria Müller Imboden, secrétaire générale

\section{Union Européenne des Médecins Omnipraticiens (UEMO)}

L'UEMO a tenu ses assises à Giardino Naxos, en Sicile, et à Florence. La formation postgraduée en médecine générale, la défense des intérêts de l'UEMO à Bruxelles ainsi que les questions financières ont été les thèmes principaux de ces rencontres. L'UEMO s'est prononcée clairement pour une durée de la formation postgraduée de cinq ans et l'admission de la médecine générale (médecine de premier recours) en tant que spécialité dans les titres III de la directive européenne 93/16. Le style adopté pour pratiquer ce «lobbying» dépend de la présidence qui, jusqu'en 2004, relèvera de la Suède. A fin 2002, le mandat de la vice-présidence, assumé par la Suisse en la personne du Dr Harmut Seifert, Meilen, est arrivé à échéance. Pour l'heure, la délégation suisse à l'UEMO est composée des Drs Daniel Widmer (chef de délégation), Lausanne, et Reto Laetsch, Bad Ragaz.

Dr Reto Laetsch

\section{Union Européenne des Médecins Spécialistes (UEMS)}

L'UEMS s'occupe de la structuration et de l'harmonisation de la formation postgraduée des médecins et depuis quelques années aussi de leur formation continue. Bien que la Suisse ne fasse pas partie de l'UE, la FMH est depuis 1990 membre à part entière de cette organisation, dont le Dr René Salzberg a assumé la vice-présidence de 1999 à 2002.

Le Conseil de direction de l'UEMS, organe directeur de cette organisation, se réunit deux fois par an pour prendre ses décisions.

Le congrès réunissant quelque 100 délégués avec leurs accompagnants s'est déroulé à Stockholm (Suède) en 2002. A cette occasion, dans le cadre du renouvellement du Comité exécutif, le Dr Hannu HALILA (Finlande) a été élu à la présidence de l'UEMS. 


\section{Association Européenne des Médecins des Hôpitaux (AEMH)}

L'Association européenne des médecins des hôpitaux s'est réunie les 6 et 7 septembre dernier à Berlin. Seuls quelques éléments d'intérêt pratique direct ont été traités, soit la question du risque médical où un groupe de travail est à l'œuvre et les conséquences de la décision des Cours de justice européennes du 3 octobre 2000 en matière de conditions de travail et de gardes. En effet à la suite d'un litige dans la Province de Valence (Espagne), les Cours européennes ont été amenées à recommander un horaire de travail de $48 \mathrm{~h}$ par semaine pour les médecins-chefs et $11 \mathrm{~h}$ de repos après une nuit de garde entière. L'AEMH est consciente que cela entraîne des coûts supplémentaires significatifs en même temps que des pénuries probables de collaborateurs. Elle souhaite néanmoins que les moyens soient dégagés progressivement pour mettre en place ces nouveaux éléments de la directive européenne sur les heures de travail et de manière à ne pas nuire ni à la qualité ni à la formation médicale. Un dernier groupe de travail animé par la délégation suisse doit se livrer à une enquête sur le rôle des médecins-chefs dans le management et en dégager des propositions.

L'AEMH a surtout été préoccupée des réformes structurelles à mettre en œuvre dans les organisations médicales européennes pour se faire entendre d'une seule voix à Bruxelles. Une réforme est en cours pour désigner la CPME (Comité permanent des médecins européens) comme instance faîtière regroupant les différentes autres associations existantes, médecins omnipraticiens, spécialistes, salariés, assistants, etc. L'élargissement de l'Union européenne et la mise en place d'une constitution font à ce titre l'objet de vives préoccupations afin d'éviter des disparités avec les futurs états-membres, de noyer la santé dans le vaste domaine de l'assurance sociale sans plus, et de garantir la qualité et l'égalité d'accès. La première phase d'élargissement en 2004 et la deuxième en 2007 font surgir toute une série de problèmes pratiques majeurs, en particulier dans le domaine de la libre circulation des personnes), qui ne peuvent laisser la Suisse indifférente. N'ayant aucun moyen d'influencer les décisions au niveau politique, bien qu'elles nous concernent tout aussi directement par le biais des bilatérales, il nous reste nos organisations professionnelles. La FMH n'est pour le moment pas membre de la CPME pour des raisons essentiellement budgétaires (Fr. 45 000.+ frais de représentation dont une place à mitemps au moins). Il va devenir sans doute impératif de faire un effort supplémentaire pour le de- venir à bref délai ou alors accepter d'exécuter et de se taire au nom de «l'indépendance» comme c'est le cas dans bien d'autres domaines ...

Dr Yves Guisan

\section{European Forum of Medical Associations and WHO (EFMA / WHO)}

Le Forum européen des associations médicales/ OMS, dans le comité duquel la FMH est représentée depuis sa fondation en 1984, a tenu sa séance annuelle à Vienne en avril 2002 à l'invitation de la Chambre médicale autrichienne. 34 associations médicales nationales européennes y ont participé ainsi que de nombreux représentants de l'Organisation mondiale de la santé. Les thèmes de cette rencontre ont été les suivants: formation continue des médecins en Europe, rapports des organisations médicales sur les modifications de leur système national de santé, exposés sur la médecine factuelle et réapparition d'anciennes maladies infectieuses, telles la tuberculose, considérées à tort comme éradiquées.

La FMH assume l'infrastructure de la logistique du Forum jusqu'à nouvel avis. Le Dr René Salzberg y siège au titre de secrétaire général honoraire.

La séance 2003 de l’EFMA/WHO est prévue en février à Berlin.

Dr René Salzberg

\section{Association Médicale Mondiale (World Medical Association)}

L'Association médicale mondiale, dont la FMH est à nouveau membre à part entière depuis l'année dernière, devient de plus en plus un forum planétaire pour les associations médicales nationales et les organisations internationales, telles que l'OMS ou diverses ONG. Elles y discutent de sujets d'éthique médicale et y formulent des directives aussi bien théoriques que pratiques. Les rencontres de cette année à Divonne (F) et Washington, D.C. (USA) ont abordé avant tout la problématique des expérimentations cliniques sur l'être humain (révision de la déclaration $\mathrm{d}^{\prime}$ Helsinki) et des questions de bio-terrorisme depuis les attentats du 11 septembre 2001 à New York et Washington. Un symposium scientifique de deux jours a eu lieu à Washington avec des scientifiques et des politiciens en provenance essentiellement des USA. La doctoresse Myllimäkki de Finlande a été élue présidente de l'Association.

Dr René Salzberg 


\section{Secrétariat général}

\section{Quelle année!}

2002 a été une année pleine de turbulences pour la FMH. Il a fallu organiser la votation générale sur le TARMED, décidée par la Chambre médicale extraordinaire à fin 2001. Cette votation, dont le déroulement précis est fixé dans les statuts mais qui avait rarement été organisée, s'est déroulée fort heureusement sans grandes difficultés. Malgré les efforts d'économies, ce scrutin a coûté à la FMH la coquette somme de Fr. 284 567.-.

La date de la Chambre médicale ordinaire, fixée tôt dans l'année, a exercé une pression sur la clôture des comptes annuels et le processus de budgétisation. On s'est rapidement aperçu que les tâches prévues ne pourraient pas être réalisées avec les moyens à disposition. Aussi, ce fut un grand soulagement d'apprendre que la Chambre médicale avait décidé d'augmenter la cotisation ordinaire de membre de $10 \%$. Le financement de notre Fédération nationale est ainsi assuré à moyen terme. Les dépenses pour des activités relatives à la suppression éventuelle de l'obligation de contracter n'ont cependant pas (encore) été prises en compte dans ce contexte.

L'entrée en vigueur des accords bilatéraux avec l'Union européenne en début juin 2002 a modifié le statut de la FMH de manière indirecte mais durable: l'obligation de s'affilier n'existe plus. Le scénario possible mais invraisemblable d'un exode massif des membres n'a pas eu lieu. Au contraire! La décision intempestive du Conseil fédéral, un mois plus tard, d'introduire un blocage de l'accès la pratique privée pour les jeunes médecins, a conduit à une augmentation du nombre de nouveaux membres.

Par la même occasion, le blocage de l'accès à la pratique privée a occasionné un travail supplémentaire important pour le secrétariat général. Alors que les demandes de renseignements et de conseils dans les domaines juridiques et pratiques ont diminué après quelques semaines, les demandes de titres sont restées très nombreuses jusqu'à la fin de l'année. Comme elles étaient formulées pour des titres obtenus selon l'ancien droit et qu'elles ne tombaient donc pas sous le coup du nouveau tarif des émoluments couvrant les frais, les recettes du centre de coûts FPPC sont restées bien en deçà des prévisions.

Les discussions sur le tarif médical ont diminué fortement après les résultats positifs de la vo- tation générale. Les bulletins TARMED-Info, lancés en mai puis publiés chaque mois dans le Bulletin des médecins suisses, se sont avérés très constructifs. Les améliorations de reengineering effectuées en cours d'année ont été accomplies dans le cadre d'un travail intensif mais sans à-coup. Les préparatifs pour le recensement de la valeur intrinsèque prévu en 2003 ont également occasionné des coûts élevés. Néanmoins, il a été possible d'économiser $42 \%$ par rapport à l'année précédente pour le projet TARMED.

Les différents services d'état-major, comme la prévention, l'assurance-qualité, l'informatique médicale, etc. ont poursuivi leurs activités avec discrétion mais efficacité. Les projets d'une certaine importance - tel le remplacement des logiciels de la FMH - n'ont pas pu être réalisés par manque de capacités durant l'exercice passé sous revue, ce qui a détendu la situation financière.

\section{Effectif du personnel}

L'année 2002 a montré que l'on ne pouvait pas augmenter indéfiniment la production. Malgré le renfort de 6 nouveaux collaborateurs, soit 445 pour cent de postes, et l'engagement de personnel temporaire, la charge de travail des collaborateurs est restée très élevée, aux confins du supportable. Les mouvements de personnel ont augmenté en conséquence. Fort heureusement, les collaborateurs ont conservé leur motivation et leur plaisir à travailler. Nous souhaitons vivement qu'il en aille de même ces prochaines années. Des collaborateurs connaissant bien leur domaine d'activité et hautement motivés sont notre capital le plus précieux et nous devons en prendre soin!

Ont fêté un anniversaire de service:

- Ueli Bucher, informatique (15 ans);

- Robert «Röbi» Gmür, service juridique (15 ans),

- Barbara Linder, service juridique/MPA (10 ans),

- Barbara Weil, prévention (10 ans).

Ont quitté le secrétariat général:

- Severine Allimann, bureau d'expertises extrajudiciaires/SRIM Lausanne;

- Franca Bodenmann, registre des membres;

- Mario Clément, informatique;

- Marco Fachin, chef du service de traduction; 
- Christina Nyffeler, assistantes médicales / documentation;

- Ruth Schindler, registre des membres.

Ont été engagés:

- Katrin Brülhart, FPPC;

- Michael Buletti, secrétariat de direction;

- Annelise Bütschi-Grünig, FPPC;

- Sabina Carulli, registre des membres;

- Jasmine Catalano, FPPC;

- Martin Denz, informatique médicale/ télémédecine;

- Natalie Favre, service juridique/bureau d'expertises extrajudiciaires;

- Monika Kölliker, secrétariat de direction;

- Diane Redard, bureau d'expertises extrajudiciaires / SRIM Lausanne;

- Simon Ryter, informatique;

- Claude Noëlle Schmied, assistantes médicales / documentation;

- Augustin Wyss, chef du service de traduction.

Je tiens à exprimer ma vive gratitude à tous nos collaborateurs pour leur engagement hors du commun.

Je saisis également l'occasion de remercier personnellement tous ceux qui m'ont soutenue avec dynamisme et compréhension dans ma première année de service comme secrétaire générale et qui ont facilité mon démarrage.

Annamaria Müller Imboden, secrétaire générale

\section{Bilan}

Au cours de l'exercice écoulé, la somme du bilan a augmenté de 5,7\%. Cette année également, la situation des marchés financiers a incité à la prudence en matière de placements. L'augmentation des actifs est due essentiellement à l'augmentation des liquidités.
Concernant les passifs, on enregistre une réduction des dettes à court terme envers des organisations liées. Les capitaux étrangers à long terme ont augmenté malgré le remboursement de l'hypothèque immobilière au moyen des sommes dégagées par les obligations parvenues à terme, car il a fallu augmenter massivement les provisions à la suite des estimations négatives concernant les prêts et participations et pour pouvoir alimenter le fonds pour les pensions de retraite.

\section{Compte d'exploitation}

Le compte d'exploitation dépasse d'à peine un million de francs celui de l'année précédente, mais se situe toutefois bien en-deçà des attentes. Le plan financier nécessite d'importants excédents cette année et l'année prochaine de manière à pouvoir couvrir les déficits prévus dans le domaine de la formation prégraduée, postgraduée et continue pour les exercices suivants. La planification avait été basée sur une facturation d'après le nouveau tarif des émoluments dès le début de l'année, laquelle a été retardée.

Les recettes supplémentaires du centre de coûts FPPC, à hauteur de 1,5 millions de francs en chiffre rond au lieu des 3,2 millions de francs budgétisés, ont été balancées pour un tiers par des dépenses supplémentaires. Malgré des économies sur les dépenses directes et une politique salariale stricte, l'énorme charge de travail, due en particulier à la votation générale, à l'entrée en vigueur de la LEPM et au blocage de l'accès à la pratique privée, a engendré des dépenses supplémentaires d'environ 0,5 million de francs.

La FMH enregistre des recettes liées en provenance de projets ou d'activités pour un total d'environ 3,4 millions de francs. Comme ces fonds sont comptabilisés par la FMH, ils doivent être attestés dans les comptes selon les directives ad hoc. 
Tableau 1

Bilan au 31 décembre 2002 (comparaison avec l'année précédente).

\section{Désignation}

\section{ACTIFS}

Capital de roulement

Liquidités

Titres et valeurs

Total des liquidités et des titres et valeurs

Créances sur prestations

Autres créances

Total des créances

Actifs de régularisation

Total des actifs de régularisation

Total du capital de roulement

Capital investi

Participations

Créances à long terme envers des entreprises liées

Total des investissements financiers

Mobilier et équipement

Machines de bureau, install. informatique, logiciels

Biens immobiliers

Total des biens

Total du capital investi

Total des ACTIFS

\section{PASSIFS}

Capitaux étrangers

Dettes à court terme

Dettes résultant de projets en cours

Passifs de régularisation

Total des capitaux étrangers à court terme

Charge financière à long terme

Provisions pour réseau

Provisions pour participations

Provision pour votation générale

Provisions pour frais de personnel

Autres provisions

Total des capitaux étrangers à long terme

Capital effectif

Capital

Bénéfice du bilan

Total du capital effectif

Total des PASSIFS
2002

2001

Fr.

Fr.

5902583.04

5452809.70

1121011.25

7023594.29

2048906.24

6814.20

2055720.44

7815.45

7815.45

9087130.18

914500.00

897082.00

1811582.00

40000.00

345000.00

1396800.00

1781800.00

3593382.00

12680512.18

1143495.25

6596304.95

1977873.75

28298.35

2006172.10

11556.20

11556.20

8614033.25

889500.00

817082.00

1706582.00

37000.00

239500.00

1396800.00

1673300.00

3379882.00

11993915.25

\begin{tabular}{rr}
2028845.66 & 2739154.75 \\
629003.01 & 705375.53 \\
336953.25 & 426460.75 \\
2994801.92 & 3870991.03 \\
\hline 0.00 & 445000.00 \\
100000.00 & 100000.00 \\
1312582.00 & 776850.00 \\
0.00 & 150000.00 \\
1762400.00 & 1153900.00 \\
850000.00 & 850000.00 \\
4024982.00 & $\underline{3475750.00}$ \\
\hline 4647174.22 & \\
1013554.04 & 4595170.31 \\
\hline $\mathbf{5 6 6 0 7 2 8 . 2 6}$ & 52003.91 \\
\hline 12680512.18 & $\underline{4647174.22}$ \\
\hline
\end{tabular}


Tableau 2

Compte d'exploitation du $1^{\text {er }}$ janvier au 31 décembre 2002 (comparaison avec l'année précédente).

\section{Désignation \\ PRODUITS \\ Produits d'exploitation}

Cotisations de membres

11602911.00

4735635.26

1528283.48

Recettes provenant de projets et autres recettes

Total des produits d'exploitation

$-449074.00$

$-3979.41$

$-453053.41$

$-292094.03$

17121682.30
11303225.70

2998931.08

2017628.38

16319785.16

121485.30

17824.49

139309.79

$-719594.54$

15739500.41

Total des PRODUITS

$-336900.00$

0.00

$-1613971.19$

$-1966433.39$

$-2022127.62$

Dépenses pour projets et divers

$-5939432.20$

Total des charges directes

Frais de personnel

$-7799469.56$

Autres frais d'exploitation

Frais de locaux

$-190722.51$

$-14862.60$

$-12423.08$

$-11078.14$

$-1274.36$

Frais d'énergie et taxes d'ordures

Frais d'administration et d'informatique

Frais de publicité

Autres frais d'exploitation

Résultat financier

Total des autres frais d'exploitation

Résultat avant impôts et amortissements

447318.35

$-75256.81$

$-7660.50$

47085.50

$-1713510.85$

1669269.69

Impôts et amortissements

Impôts sur bénéfice et capital

$-5629.35$

$-262140.30$

$-387946.00$

Amortissements

$-655715.65$

Total impôts et amortissements

$-16108128.26$

Total des CHARGES

1013554.04

$-1327346.48$

$-2112562.11$

$-2865278.77$

$-6305187.36$

$-7693088.94$

$-186572.68$

$-17159.08$

$-18642.02$

$-7909.88$

$-1189.85$

$-935039.63$

$-36995.78$

$-1130.37$

75495.55

$-1129143.74$

612080.37

$-24122.60$

$-258483.46$

$-277470.40$

$-560076.46$

$-15687496.50$

52003.91 